\title{
Possibilistic Decision-Making Approaches
}

\author{
Peijun Guo \\ Faculty of Business Administration, Yokohama National University, 79-4 Tokiwadai, Hodogaya-Ku, Yokohama \\ 240-8501, Japan
}

\begin{abstract}
In this paper, decision-making approaches with partially known information characterized by possibility distributions are proposed. Five kinds of criteria are provided for evaluating the alternatives. Based on the proposed possibilistic decision approaches, a newsvendor problem is analyzed.
\end{abstract}

Keywords: Decision analysis, Possibility theory, Newsvendor problem

\section{Introduction}

A decision-making situation includes several components, i.e. decision alternatives, outcomes and states of the nature. Alternatives are typically courses of action that are open to a decision-maker at the times of the decision. The effect of a decision, called outcome, depends not only on the choice of an alternative but also factors outside of the decisionmaker's control, i.e. states of nature. The possible outcomes of a decision are the combined effects of a chosen alternative and the states of nature. Decision analysis is choosing among alternatives considering information about the states of nature. As a limiting case, a decision-maker may know which state of nature will occur. In such a case (known as decision making under certainty), it is easy to make a choice because the outcome of each alternative is deterministic. Otherwise, a decision-maker acts under non-certainty. Non-certainty is divided into three categories -risk, uncertainty, and ignorance. Situations involving risk are when the probabilities of all possible outcomes in the future can be exactly calculated. On the other hand, uncertain situations are associated with partially known information when probabilities cannot be obtained exactly [21]. Situations involving ignorance occurs when no information is available to distinguish which state of nature is more likely to happen. Possibility theory, initiated by Zadeh [34] and advanced by Dubois, Prade and Klir [2], [20] is one of the current uncertainty theories devoted to handing of incomplete information in the real world. Possibility theory is based on two basic non-additive measures, possibility measure and necessity measure. Just as probabilities can be interpreted in many ways (e.g., frequentist view and subjective view), possibilities can be explained from several semantic aspects. One way to explain possibility is feasibility, such as ease of achievement [34]. Another way to explain is plausibility, which refers to the propensity of events to occur, which relates to the concept "potential surprise" [30]. Third way to explain possibility is logical consistency with available information [28], [33]. Finally possibility can be explained as preference, referring to the willingness of agent to make a decision [3]. Necessity is dually related to possibility in sense that "not A" being not possible means that $\mathrm{A}$ is necessary. A semantic analysis can be done in parallel with possibility, referring to belief, acceptance and priority. Decision analysis based on possibility theory has been researched in the papers [5]-[12], [31]-[32].

In this paper, decision-making approaches with partially known information characterized by possibility distributions are proposed. Five kinds of criteria are provided for evaluating the alternatives. Based on the proposed possibilistic decision approaches, a newsvendor problem is analyzed.

\section{Possibilistic decision criteria}

The first step in the decision analysis is problem formulation. The set of an alternative $a$ is denoted as $A=\{a\}$. The uncertain future event is referred to as chance event and its outcomes are referred to as the states of nature. Denoting a state of nature as $x$, the set of the states of nature is $S=\{x\}$. The consequence resulting from a specific combination of an alternative $a$ and a state of nature $x$ is refereed to as a payoff, denoted as $p(x, a)$. A normalized function $u(x, a)$ defined on $\left[x_{l}, x_{u}\right]$ is called a satisfaction function, that expresses the satisfaction level of a decision maker for the payoff $p(x, a)$. The degree of which state of nature being more possible to happen in the future can be characterized by a possibility distribution, defined as follows.

Definition 1. Given a function

$$
\pi: S \rightarrow[0,1]
$$




$$
\max _{x \in S} \pi(x)=1
$$

then the function $\pi(x)$ is called the possibility distribution and the value of $\pi(x)$ is called the possibility degree of $x \cdot \pi(x)=1$ means that it is normal that the state of nature $x$ occurs and $\pi(x)=0$ means that it is abnormal that $x$ occurs. The smaller the possibility degree of $x$, the more surprised the happening of $x$. The possibility distribution can be used to represent the knowledge or judgment of experts. Several kinds of methods for identifying possibility distributions have been proposed [9], [11], [12].

The possibility distribution determines the unique possibility and necessity measures of a chance event $\mathrm{E}$, denoted as $\operatorname{Pos}(E)$ and $\operatorname{Nec}(E)$ via the following formulas

$$
\begin{aligned}
& \operatorname{Pos}(E)=\max _{x \in E \subseteq S} \pi(x), \\
& \operatorname{Nec}(E)=1-\sup _{x \in E^{c} \subseteq S} \pi(x), \\
& \operatorname{Pos}(\{x\})=\pi(x) \quad x \in S .
\end{aligned}
$$

An alternative $a$ can be evaluated according to the following criteria.

Criterion 1: $C_{1}(a)=\int_{0}^{1} r P o s(x \mid u(x, a) \geq r) d r$,

which is a possibility measure-based decision criterion. Criterion 2: $C_{2}(a)=\int_{0}^{1} r N e c(x \mid u(x, a) \geq r) d r$,

which is a necessity measure-based decision criterion.

Criterion 3: $C_{3}(a)=\int_{x_{l}}^{x_{u}} u(x, a) \cdot \pi(x) d x$,

where the possibility level is regarded as the weight of the satisfaction.

Criterion 4: $C_{4}(a)=\int_{x_{l}}^{x_{u}} \max (u(x, a), \pi(x)) d x,(9)$ which is an optimistic decision criterion.

Criterion 5: $\quad C_{5}(a)=\int_{x_{l}}^{x_{u}} \min (u(x, a), \pi(x)) d x$,

which is a pessimistic decision criterion.

Clearly, the following relations hold.

$$
\begin{aligned}
& C_{4}(a)>C_{5}(a)>C_{3} \\
& C_{1}(a)>C_{2}(a)
\end{aligned}
$$

Theorem 1. For the given possibility distribution, if $u\left(x, a_{1}\right) \geq u\left(x, a_{2}\right)$ holds, then we have

(I) $C_{1}\left(a_{1}\right) \geq C_{1}\left(a_{2}\right)$

(II) $C_{2}\left(a_{1}\right) \geq C_{2}\left(a_{2}\right)$

(III) $C_{3}\left(a_{1}\right) \geq C_{3}\left(a_{2}\right)$

(IV) $C_{4}\left(a_{1}\right) \geq C_{4}\left(a_{2}\right)$
(V) $C_{5}\left(a_{1}\right) \geq C_{5}\left(a_{2}\right)$

Proof. Obviously, (III)-(V) hold with considering (8)(10). $u\left(x, a_{1}\right) \geq u\left(x, a_{2}\right) \quad$ leads to $\left\{x \mid u\left(x, a_{1}\right) \geq r\right\} \supseteq\left\{x \mid u\left(x, a_{2}\right) \geq r\right\}$ holds for any $r$. So that we have

$\operatorname{Pos}\left(x \mid u\left(x, a_{1}\right) \geq r\right) \geq \operatorname{Pos}\left(x \mid u\left(x, a_{2}\right) \geq r\right)$,

$\operatorname{Nec}\left(x \mid u\left(x, a_{1}\right) \geq r\right) \geq \operatorname{Nec}\left(x \mid u\left(x, a_{2}\right) \geq r\right)$.

As a result, (I) and (II) hold.

$a_{1}$ is

Definition 2. For two alternatives $a_{1}$ and $a_{2}, a_{1}$ is indifferent to $a_{2}$ according to criterion $i(i=1, \ldots, 5)$ if and only if $C_{i}\left(a_{1}\right)=C_{i}\left(a_{2}\right)$ holds. $a_{1}$ is weakly preferred to $a_{2}$ according to criterion $\mathrm{i}(i=1, \ldots, 5)$ if and only if $C_{i}\left(a_{1}\right) \geq C_{i}\left(a_{2}\right)$ holds.

It can be seen that the preference relations defined in Definition 1 comply with completeness, transitivity.

Assume that the set of states of nature is a finite set $X=\left\{x_{1}, \cdots x_{n}\right\}$, then (6)-(10) become the following formula.

Criterion 1:

$$
\begin{aligned}
& R_{1}(a) \\
& =\sum_{x_{i}} u\left(x_{i}, a\right) \max _{x_{j}}\left(\pi\left(x_{j}\right) \mid u\left(x_{j}, a\right) \geq u\left(x_{i}, a\right)\right)
\end{aligned}
$$

Criterion 2:

$$
\begin{aligned}
& R_{2}(a)=u\left(x^{*}, a\right) \\
& +\sum_{x_{i} \in X-\left\{x^{*}\right\}} u\left(x_{i}, a\right)\left(1-\max _{x_{j}}\left(\pi\left(x_{j}\right) \mid u\left(x_{j}, a\right)<u\left(x_{i}, a\right)\right)\right)
\end{aligned}
$$

where

$$
x^{*}=\arg \min _{x_{i}} u\left(x_{i}, d\right) .
$$

Criterion 3: $R_{3}(a)=\sum_{x_{i}} u\left(x_{i}, a\right) \pi\left(x_{i}\right)$.

Criterion 4: $R_{4}(a)=\sum_{x_{i}} \max \left(u\left(x_{i}, a\right), \pi\left(x_{i}\right)\right)$.

Criterion 5: $R_{5}(a)=\sum_{x_{i}} \min \left(u\left(x_{i}, a\right), \pi\left(x_{i}\right)\right)$.

According to the Definition 1, the optimal alternative $a^{*}$ can be obtained as follows.

$$
\begin{gathered}
a^{*}=\arg \max _{a} R_{i}(a) \text { or } a^{*}=\arg \max _{a} C_{i}(a) \\
(i=1, \ldots, 5)
\end{gathered}
$$

In what follows, let us consider how to determine the optimal order in newsvendor problems according to the proposed decision criteria. 


\section{Newsvendor problems}

The newsvendor problem, also known as newsboy or single-period problem is a common inventory management problem. In general, the newsvendor problem has the following characteristics. Prior to the season, the buyer must decide what quantity of the goods to purchase/produce. The procurement leadtime tends to be quite long relative to the selling season, so the buyer can not observe demand prior to placing the order. Due to the long lead-time, often there is no opportunity to replenish inventory once the season has begun. Excess stock can only be salvaged at loss once the season is over. As well known that newsvendor problem derives its name from a common problem faced by a person selling newspapers on the street, interest in such a problem has increased over the past 40 years partially because of the increased dominance of service industries for which newsvendor problem is very applicable in both retailing and service organizations. Also, the reduction in product life cycles makes newsvendor problem more relevant. Many extensions have been made in the last decade, such as different objects and utility functions, different supplier pricing policies, different new-vendor pricing policies [19], [24], [27]. Almost all of extensions have been made in the probabilistic framework; that is, the uncertainty of demand and supply is characterized by the probability distribution, and the objective function is expressed as maximizing the expected profit or probability measure of achieving a target profit. Some papers have dealt inventory problems using fuzzy sets theory [4], [15]-[18], [23], [25], [29]. However, there are few papers dealing with the uncertainty in newsvendor problems by fuzzy methods. Buckley [1] used possibility distribution to represents a decisionmaker's linguistic expression on demand, such as "good" and "not good" etc., and introduced some fuzzy goal to express decision-maker's satisfaction. The order quantity was obtained based on possibility and necessity measures, which made the possibility of not achieving fuzzy goal was sufficiently low and achieving fuzzy goal was sufficiently high. Ishi et al. [13] investigated the fuzzy newsvendor problem in which the shortage cost was vague and expressed as a fuzzy number. An optimal order quantity was obtained by fuzzy maximal order. Petrovic et al. [26] gave a fuzzy newsvendor model where uncertain demand was represented by a group of fuzzy sets and inventory cost was given by a fuzzy number. The defuzzification method was used to obtain an optimal order quantity. Kao et al. [14] obtained the optimal order quantity to minimize the fuzzy cost by comparing the area of fuzzy numbers. Li et al. [22] proposed two models, in one the demand was probabilistic while the cost components were fuzzy and in the other the costs were deterministic but the demand was fuzzy. The profit was maximized through ordering fuzzy numbers with respect to their total integral values. Guo [5] proposed some new newsvendor models with possibilistic information.

In this paper, new newsvendor models are proposed with the emphasis that the products are of short life-cycle which lack prior information on their demands, such as fashion goods, where no data can be used for statistical analysis to predict the coming demand. The uncertainty of demand is characterized by the possibility distribution instead of fuzzy membership function. In other words, the uncertain demand is not described by the fuzzy linguistic language, such as "good", "better" etc., to only reflect the subjective judgment of decision-maker. The uncertain demand is characterized from the viewpoint of measure theory where the possibility degree reflect the potential of a demand happening. The other should be noted is that the optimal order is determined according to the proposed five criteria to totally evaluate the alternative from satisfaction and plausibility aspects instead of maximizing mean value in probabilistic models and ranking fuzzy numbers in fuzzy models.

Consider a retailer who sells a short life cycle, or single-period new product. The retailer orders $q$ units before the season at the unit wholesale price $W$. When demand $x$ is observed, the retailer sell units (limited by the supply $q$ and the demand $x$ ) at unit revenue $R$ with $R>W$. Any excess units can be salvaged at unit salvage price $S_{o}$ with $W>S_{o}$. If shortage, the lost chance price is $S_{u}$. The profit function of the retailer is as follows:

$r(x, q)=\left\{\begin{array}{l}R x+S_{o}(q-x)-W q ; \text { if } x<q \\ (R-W) q-S_{u}(x-q) ; \text { if } x \geq q\end{array}\right.$

Definition 2. The satisfaction function of a retailer is defined by the following strictly increasing function of profit $r$,

$$
u:\left[r_{l}, r_{u}\right] \rightarrow[0,1]
$$

where $r_{l}$ and $r_{u}$ are the lowest and highest profit, respectively, $u\left(r_{l}\right)=0$ and $u\left(r_{u}\right)=1$.

In what follows, we give a numerical example to explain how to make decision based on the proposed five criteria.

The unit wholesale price $W$, the unit revenue $R$, the unit salvage price $S_{o}$ and the lost chance price $S_{u}$ are $60 \$, 100 \$, 20 \$$ and $50 \$$, respectively. Based on his considerable experience, he gives some estimate, presented by the possibilities of demand as listed in Table 1. 


\begin{tabular}{|c|c|c|c|c|c|c|}
\hline demand & 5 & 6 & 7 & 8 & 9 & 10 \\
\hline possibilities & 0.2 & 0.5 & 0.8 & 1 & 0.8 & 0.5 \\
\hline
\end{tabular}

Table. 1: The possibilities of demand.

According to (26), the profit matrix is obtained as shown in Table 2.

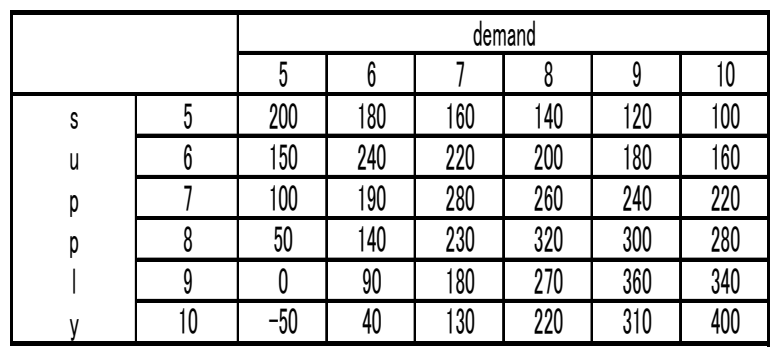

Table. 2: The profit matrix.

The satisfaction function defined on $[-50,400]$ is given as

$$
u(r)=(r+50) / 450,
$$

and the satisfaction matrix is shown in Table 3.

\begin{tabular}{|c|c|c|c|c|c|c|c|}
\hline \multicolumn{2}{|c|}{} & \multicolumn{6}{|c|}{ demand } \\
\cline { 2 - 8 } \multicolumn{2}{c|}{} & 5 & 6 & 7 & 8 & 9 & 10 \\
\hline \multirow{3}{*}{$s$} & 5 & 0.55556 & 0.51111 & 0.46667 & 0.42222 & 0.37778 & 0.33333 \\
\cline { 2 - 8 } & 6 & 0.44444 & 0.64444 & 0.6 & 0.55556 & 0.51111 & 0.46667 \\
\cline { 2 - 8 } & 7 & 0.33333 & 0.53333 & 0.73333 & 0.68889 & 0.64444 & 0.6 \\
\cline { 2 - 8 } & 8 & 0.22222 & 0.42222 & 0.62222 & 0.82222 & 0.77778 & 0.73333 \\
\hline & 9 & 0.11111 & 0.31111 & 0.51111 & 0.71111 & 0.91111 & 0.86667 \\
\cline { 2 - 8 } & 10 & 0 & 0.2 & 0.4 & 0.6 & 0.8 & 1 \\
\hline
\end{tabular}

Table. 3: The satisfaction matrix.

Using (20)-(24), $R_{i}(a)$ ( $\left.a=5, \ldots, 10 ; i=1, \ldots, 5\right)$ are obtained as shown in Table 4. Using (28), the obtained optimal orders according to Criterion 1 to 5 are $8,7,8$, 10,8 , respectively.

\begin{tabular}{|c|c|c|c|c|c|c|}
\hline \multicolumn{2}{|c|}{} & \multicolumn{5}{|c|}{ Criteria } \\
\cline { 3 - 7 } \multicolumn{2}{c|}{} & 1 & 2 & 3 & 4 & 5 \\
\hline \multirow{3}{*}{$\mathrm{s}$} & 5 & 1.87299 & 0.60663 & 1.63111 & 4.16667 & 2.3 \\
\cline { 2 - 7 } $\mathrm{u}$ & 6 & 2.78 & 1.18444 & 2.08889 & 4.18889 & 2.83333 \\
\cline { 2 - 7 } $\mathrm{p}$ & 7 & 3.38632 & 1.51999 & 2.42444 & 4.06667 & 3.26667 \\
\cline { 2 - 7 } $\mathrm{p}$ & 8 & 3.59999 & 1.33777 & 2.56444 & 4.05556 & 3.34444 \\
\cline { 2 - 7 } & 9 & 3.06666 & 0.75778 & 2.46 & 4.27778 & 2.94444 \\
\cline { 2 - 7 } $\mathrm{v}$ & 10 & 2.34 & 0.36 & 2.16 & 4.3 & 2.5 \\
\hline
\end{tabular}

Table. 4: The value of $R_{i}(a)$.

\section{Conclusions}

In this paper, decision-making approaches with partially known information characterized by possibility distributions are proposed. Five kinds of criteria are provided for evaluating the alternatives. Based on the proposed possibilistic decision approaches, a newsvendor problem is analyzed. The different decision criteria lead to the different optimal orders that reflect the various attitudes on satisfaction and plausibility. It can be seen that possibilistic decision models are very effective especially for the cases of incomplete information, ignorance, or high cost of information acquisition because uncertainty can be characterized by possibility distributions.

\section{References}

[1] J.J. Buckley, Possibility and necessity in optimization. Fuzzy Sets and Systems, 25: 1-13, 1988.

[2] D. Dubois and H. Prade, Possibility Theory, Plenum Press, New York, 1988.

[3] D. Dubois and H. Prade, Possibility theory as a basis for qualitative decision theory. In Proceedings of the $14^{\text {th }}$ International Joint Conference on Artificial Intelligence, pp. 19241930, 1995.

[4] M. Gen, Y. Tsujimura, D. Zheng, An application of fuzzy set theory to inventory control models. Computers ind. Engng, 33: 553-556, 1997.

[5] P. Guo, Newsvendor problem based possibility theory, Lecture Notes in Computer Science. Proceeding of 7th International Conference of Knowledge-Based Intelligent Information and Engineering Systems, Oxford, UK, 2773: 213219, 2003.

[6] P. Guo, Decision analysis based on active focus point and passive focus point. Proceedings of the International Workshop of Fuzzy Systems and Innovational Computing, pp. 39-44, 2004.

[7] P. Guo, Urban land development based on possibility theory. Proceedings of 2004 Annual Meeting of NAFIPS, North American Fuzzy Information Processing Society, pp. 384-389, 2004.

[8] P. Guo, H. Tanaka, M. Inuiguchi, Selforganizing fuzzy aggregation models to rank the objects with multiple attributes. IEEE Transactions on SMC, Part A: Systems and Humans, 30: 573-580, 2000.

[9] P. Guo, H. Tanaka and H. -J. Zimmermann, Upper and lower possibility distributions of fuzzy decision variables in upper level decision problems. Fuzzy Sets and Systems, 111: 71-79 2000.

[10] P. Guo and H. Tanaka, Fuzzy DEA: A perceptual evaluation method. Fuzzy Sets and Systems, 119: 149-160, 2001. 
[11] P. Guo, D.Zeng, H.Shishido, Group decision with inconsistent knowledge. IEEE Transactions on SMC, Part A, 32: 670-679, 2002.

[12] P. Guo and H. Tanaka, Decision analysis based on fused double exponential possibility distributions, European Journal of Operational Research 148 (2003) 467-479.

[13] H. Ishii and T. Konno, A stochastic inventory problem with fuzzy shortage cost. European Journal of Operational Research, 106: 90-94, 1998.

[14] C. Kao and W. Hsu, A single-period inventory model with fuzzy demand. Computers and Mathematics with Applications, 43: 841-848, 2002.

[15] H. Katagiri and H. Ishii, Some inventory problems with fuzzy shortage cost. Fuzzy Sets and Systems, 111: 87-97, 2000.

[16] H. Katagiri and H. Ishii, Fuzzy inventory problem for perishable commodities. European Journal of Operational Research, 138: 545-553, 2002.

[17] H. Lee and J. Yao, Economic order quantity in fuzzy sense for inventory without backorder model. Fuzzy Sets and Systems, 105: 13-31, 1999.

[18] P. Kacpryzk and P. Staniewski, Long-term inventory policy-making through fuzzy decisionmaking models. Fuzzy Sets and Systems, 8: 117 132,1982

[19] M. Khouja, The single-period (news-vendor) Problem: literature review and suggestion for future research. Omega, 27: 537-553, 1999.

[20] G.J. Klir, T. Folger, Fuzzy Sets, Uncertainty and Information, Prentice-Hall, New Jersey, 1988.

[21] F.H. Knight, Risk, Uncertainty and Profit, Harper and Row, New York, 1965.

[22] L. Li, S.N. Kabadi, K.P.K. Nair, Fuzzy models for single-period inventory problem. Fuzzy sets and Systems, 132: 273-289, 2002.

[23] D. Lin and J. Yao, Fuzzy economic production for inventory. Fuzzy Sets and Systems, 111: 465495, 2000.

[24] S.A. Lippman and K.F. McCardle, The competitive newsboy. Operations Research, 45: 54-65, 1997.

[25] K.S. Park, Fuzzy set theoretic interpretation of economic order quantity. IEEE Trans. Systems Man Cybernet. SMC, 17(6): 1082-1084, 1996.

[26] D. Petrovic, R. Petrovic and M. Vujosevic, Fuzzy model for newsboy problem, Internat. $J$. Prod. Econom, 45: 435-441, 1996.

[27] E. L. Porteus, Stochastic Inventory Theory, Handbooks in OR and MS, Vol. 2 Heyman D. P. and Sobel, M.J. eds. Elsevier Science Publisher, 605-652.
[28] H. Prade, Modal semantics and fuzzy set theory. In Recent Developments in Fuzzy Set and Possibility Theory, R. R. Yager, Ed. 232-246. Pergamon Press, New York, 1982.

[29] T.K. Roy and M. Maiti, A fuzzy EOQ model with demand-dependent unit cost under limited storage capacity. European Journal of Operational Research, 99: 425-432, 1997.

[30] G.L.S. Shackle, Expectation in Economics, Cambridge University Press, Cambridge, UK, 1949.

[31] H. Tanaka and P. Guo, Possibilistic Data Analysis for Operations Research, Heidelberg, New York, Physica-Verlag, 1999.

[32] H. Tanaka and P. Guo, Portfolio selection based on upper and lower exponential possibility distributions. European Journal of Operational Research, 114: 115-126, 1999.

[33] R.R. Yager, A foundation for a theory of possibility. J. Cybernetics, 10: 177-204, 1980.

[34] L. A. Zadeh, Fuzzy sets as a basis for a theory of possibility. Fuzzy Sets and Systems, 1: 3-28, 1978. 